\title{
Linear Chebyshev Approximation in the Complex Plane Using Lawson's Algorithm
}

\author{
By S. Ellacott and Jack Williams
}

\begin{abstract}
In this paper we discuss the application of Lawson's algorithm for computing best linear Chebyshev approximations to complex-valued functions. Some numerical examples are also presented.
\end{abstract}

1. Introduction and Preliminaries. In this paper we are concerned with the use of Lawson's algorithm ([4], [7] , [6] ) for computing best linear Chebyshev approximations to complex-valued functions. Also, we give some results which relate the best approximation on a compact set $A$ in the complex plane to best approximations on certain subsets of $A$. Some numerical examples are also presented including approximations by linear combinations of rationals with preassigned poles.

We consider the class $C[A]$ of complex-valued continuous functions $f$ on a compact subset $A$ of the complex plane, with $\|f\|=\max _{z \in A}|f(z)|$. We assume throughout that $A$ contains at least $n+2$ points. Let $P$ denote the finite-dimensional subspace of $C[A]$ whose basis is $\phi_{1}, \phi_{2}, \ldots, \phi_{n}$. Now let $f \in C[A]-P$; then we seek $p^{*} \in P$ for which

$$
\left\|f-p^{*}\right\| \leqslant\|f-p\| \quad \text { for all } p \in P \text {. }
$$

It is known [5, Chapter 2] that $p^{*}$ exists; and if $\left\{\phi_{i}\right\}$ forms a Chebyshev set on $A$, then $p^{*}$ is unique.

In practice, this approximation problem could occur in one of the following forms.

Problem 1. $A \equiv \bar{S}$, a simply-connected region bounded by a piecewise smooth Jordan curve $C$; the functions $f$ and $\left\{\phi_{i}\right\}$ are analytic in $S$ and continuous on $\bar{S}$. Then, from the maximum modulus theorem, $\|f\|=\max _{z \in C}|f(z)|$, so the problem is equivalent to best approximation on the boundary $C$. For example, we may wish to obtain a best polynomial approximation $\Sigma_{0}^{n} a_{r} z^{r}$ to $e^{z} \sin \pi z$ on the quadrant

$$
\bar{S}:=\{z:|z| \leqslant 1, \operatorname{Re}(z) \geqslant 0, \operatorname{Im}(z) \geqslant 0\} .
$$

Problem 2. $A:=\left\{z_{j}: j=1,2, \ldots, N\right\}$ on which we wish to approximate the values $f_{j}=f\left(z_{j}\right), j=1,2, \ldots, N$. For example, the $f$ values may correspond to a set of points on the imaginary axis and we seek a best approximation with $\phi_{r} \equiv 1 /\left(z-\alpha_{r}\right)$, $r=1,2, \ldots, n$, where $\left\{\alpha_{r}\right\}$ are preassigned points lying in the left half-plane.

2. Discretization Theory. In this paper we shall apply Lawson's algorithm to the above Problems 1 and 2. Cline (see [2]) has extended* Lawson's algorithm to 
apply to a continuum; but the algorithm we discuss here is only applicable to approximation on a finite point set. Naturally, we therefore, "discretize" Problem 1 by first replacing the boundary curve $C$ by a finite point set $C_{k}$ which is sufficiently "close" to $C$. Then we compute a best approximation $p_{k}$ to $f$ on $C_{k}$. This process can be justified on the basis of the following results, the first of which is due to Cheney [1, Chapter 3]. Cheney's result treats real approximation, but the same proof applies to the complex case.

Definition. Let $A$ be a compact subset of the complex plane; then the density of the subset $Z \subseteq A$ is given by

$$
|Z|=\max _{z \in A} \inf _{y \in Z}|y-z|
$$

Theorem 2.1 (Cheney [1]). Let $f \in C[A]-P$ and let $p_{k} \in P$ be a best approximation to $f$ on the compact subset $Z_{k} \subset A$, where $\left|Z_{k}\right| \rightarrow 0$ as $k \rightarrow \infty$; then $\lim _{k \rightarrow \infty}\left\|f-p_{k}\right\|=\left\|f-p^{*}\right\|$. If $p^{*}$ is a unique best approximation to $f$, then $\lim _{k \rightarrow \infty}\left\|p_{k}-p^{*}\right\|=0$.

As applied specifically to Problem 1, we now give a result which gives some information about the choice of subsets $Z_{k} \subset C$. Naturally, for results of this type the smoothness properties of both $f$ and the boundary curve $C$ are involved. Here it is more convenient to measure the density of subsets $Z_{k} \subset C$ in terms of the parametric representation of $C$. For many practical problems this measure of density would then be directly related to arc length. More general results for real approximation are given by Cheney [1].

TheOREM 2.2. Let $C:=\{z: z=\gamma(t), t \in[0,1], \gamma(0)=\gamma(1)\}$ be a piecewise smooth Jordan curve consisting of the smooth arcs $C_{r}, r=1,2, \ldots, M$, and let $f$ and $\left\{\phi_{i}\right\}$ be twice continuously differentiable on each arc $C_{r}$ Let $Z_{k} \equiv Z_{k}(t) \subseteq C$, $k=1,2, \ldots$, be a sequence of finite point subsets which each contain all the points of discontinuity of $d \gamma(t) / d t, t \in[0,1]$. If

$$
\left|Z_{k}\right|=\max _{\gamma\left(t_{2}\right) \in C} \min _{\gamma\left(t_{1}\right) \in Z_{k}} d\left(\gamma\left(t_{1}\right), \gamma\left(t_{2}\right)\right)
$$

satisfies $\left|Z_{k}\right| \rightarrow 0$ as $k \rightarrow \infty$, where

$$
d\left(\gamma\left(t_{1}\right), \gamma\left(t_{2}\right)\right)=\min \left\{\left|t_{1}-t_{2}\right|, 1-\left|t_{1}-t_{2}\right|\right\},
$$

then there exists a positive constant $K$ such that, for $k=1,2, \ldots$,

$$
\left\|f-p^{*}\right\|-\max _{z \in Z_{k}}\left|f(z)-p_{k}(z)\right| \leqslant K\left|Z_{k}\right|^{2},
$$

where $p_{k} \in P$ is a best approximation to $f$ on $Z_{k}$.

Proof. Consider $Z_{k} \subseteq C$ and let $h(z)=\left|f(z)-p_{k}(z)\right|^{2}$. Suppose that $p_{k}$ is not a best approximation to $f$ on $C$; then we can choose $t_{0} \in[0,1]$ for which $\left|h\left(\gamma\left(t_{0}\right)\right)\right|=$ $\left\|f-p_{k}\right\|^{2}$ and $\gamma\left(t_{0}\right) \in C-Z_{k}$. Consider now the interval $\left(t_{0}, t_{1}\right), t_{1}>t_{0}$, where $\gamma(t) \notin Z_{k}$ for all $t \in\left(t_{0}, t_{1}\right), \gamma\left(t_{1}\right) \in Z_{k}$. (For simplicity we have assumed that $t_{1}<1$, otherwise, a simple modification is required.) From the smoothness of $h$, 


$$
\begin{aligned}
\frac{d h}{d t}\left(\gamma\left(t_{0}\right)\right) & =0 \Rightarrow h\left(\gamma\left(t_{1}\right)\right) \\
& =h\left(\gamma\left(t_{0}\right)\right)+1 / 2\left(t_{1}-t_{0}\right)^{2} \frac{d^{2} h}{d t^{2}}\left(\gamma\left(t_{0}+\theta\left(t_{1}-t_{0}\right)\right)\right), \quad 0<\theta<1 .
\end{aligned}
$$

Therefore,

$$
\left\|f-p^{*}\right\|^{2} \leqslant\left\|f-p_{k}\right\|^{2} \leqslant \max _{z \in Z_{k}}\left|f(z)-p_{k}(z)\right|^{2}+1 / 2 Q_{k}\left|Z_{k}\right|^{2},
$$

where $Q_{k}=\sup \left|d^{2} h(\gamma(t))\right| d t^{2} \mid$, and the supremum is taken over $\gamma(t) \in C_{r}, r=1,2, \ldots$, $M$. But since $\left|Z_{k}\right| \rightarrow 0$ is also equivalent to the density of the sets tending to zero in the sense of Theorem 2.1, we have from the convergence of $p_{k}$ to $p^{*}$ that $Q_{k} \leqslant K_{1}$ for some $K_{1}$. Finally on dividing (2.1) by $\left\|f-p^{*}\right\|$ and noting that

$$
\max _{z \in Z_{k}}\left|f(z)-p_{k}(z)\right| /\left\|f-p^{*}\right\| \leqslant 1
$$

the result follows.

The above theorem shows, for example, that when approximating on a square $C$, the corner points should be included in $Z_{k}$. Also, in practice, the constant $K$ could be estimated with the aid of simple difference approximations to $d^{2} h / d t^{2}$ throughout each $C_{r}, r=1,2, \ldots, M$, thus indicating whether the $Z_{k}$ is sufficiently dense in $C$. (2.1) could also be used to bound $\left\|f-p_{k}\right\|$.

3. The Lawson Algorithm. We now consider exclusively approximation on a finite point set $Z$ which consists of $N$ distinct points and where now $\|f\|=$ $\max _{z \in Z}|f(z)|$. Lawson's algorithm computes a sequence of best weighted least squares approximations, which, under suitable conditions, converges to the best Chebyshev approximation to $f$ on $Z$. The relationship between least squares approximation and Chebyshev approximation can be seen most satisfactorily by appealing to the following form of a characterization theorem due to Rivlin and Shapiro [8] (also see [5] ; in fact, the theorem applies to approximation of real- or complex-valued continuous functions on a compact Hausdorff topological space).

THEOREM 3.1 ([8], [5, CHAPTER 2]). $p \in P$ is a best Chebyshev approximation to $f \in C[Z]$ if and only if there are $r$ points $z_{1}, z_{2}, \ldots, z_{r} \in Z_{0}$ and $r$ numbers $w_{1}>0, \ldots, w_{r}>0, \Sigma w_{k}=1, r \leqslant 2 n+1(r \leqslant n+1$ in the real case $)$, for which

$$
\sum_{k=1}^{r} w_{k}\left[f\left(z_{k}\right)-p\left(z_{k}\right)\right] \overline{\phi_{i}\left(z_{k}\right)}=0, \quad i=1,2, \ldots, n,
$$

where the extremal set is denoted by

$$
Z_{0}:=\{z:|f(z)-p(z)|=\|f-p\|, z \in Z\} .
$$

This result provides a description of the extremal set $Z_{0}$; and if $\left\{\phi_{i}\right\}$ is a Chebyshev set, then we have in addition $r \geqslant n+1$ [5, Chapter 2]. The theorem illustrates the fundamental difference between the real and complex case. In the real case the characterization can be achieved in terms of $n+1$ points (on which the equi-oscillation property is satisfied if $\left\{\phi_{i}\right\}$ is a Chebyshev set), whereas in the complex case the exact number of points is unknown, $n+1 \leqslant r \leqslant 2 n+1$. It is possible to devise an algorithm 
on the assumption that $r=n+1$ and this approach has been pursued in [9]; however, the method does not in general yield best approximations.

Theorem 3.1 can be viewed in a different way. From Eqs. (3.1), regarded now as the "normal equations", it follows that the best Chebyshev approximation on $Z$ is also a best weighted least squares approximation on $Z$ with suitably defined weights $w(z), \Sigma_{Z} w(z)=1$ (where zero weights have been assigned to points $z \notin Z_{0}$ ). Further, we note that the sufficiency part of the theorem can be established without the condition $r \leqslant 2 n+1$.

If the weights $\left\{w_{r}\right\}$ and points $\left\{z_{r}\right\} \subset Z_{0}$ were known, the best Chebyshev approximation could be easily obtained by solving the associated least squares problem (see Section 6). Lawson's algorithm may be regarded as an iteration scheme for computing these $\left\{w_{r}\right\}$ and $\left\{z_{r}\right\}$. However, the fact that the above theorem is so fundamental, that is, it is applicable to very general spaces, strongly suggests that any algorithm based on the result would not be particularly efficient. This seems to be the case in practice (see Section 6).

We now define the set of weight functions

$$
W:=\left\{w: Z \rightarrow R^{N} \mid \sum_{Z} w(z)=1, w(z) \geqslant 0 \text { for all } z \in Z\right\} .
$$

Lawson's algorithm consists of an iteration on $W$ which is defined as follows.

(a) Choose $w^{1} \in W$ such that $w^{1}(z)>0$ for all $z \in Z$.

(b) Set

$$
w^{k+1}(z)=\frac{w^{k}(z)\left|e^{k}(z)\right|}{\sum_{Z} w^{k}(y)\left|e^{k}(y)\right|}, \quad k=1,2, \ldots, z \in Z,
$$

where $e^{k}=f-p^{k}$ and $p^{k}$ is a best weighted least squares approximation to $f$ on $Z$, that is, $p^{k}$ minimizes $\left(\Sigma_{Z} w^{k}(z)|f(z)-p(z)|^{2}\right)^{1 / 2}$ over $p \in P$. This procedure will be referred to as the $\mathrm{L} 1$ algorithm.

We note that $w^{k+1} \in W$, but $w^{k+1}(z)=0$ is possible for some $z \in Z$. Also, the definition of the algorithm requires no condition on the basis $\left\{\phi_{i}\right\}$.

A proof of the convergence in the real case can be conveniently found in [6], in which it is necessary to assume that $\left\{\phi_{i}\right\}$ is a Chebyshev set. The proof is long (and is not easy) but can be appropriately modified to establish convergence in the complex case. Summarizing, we have, subject to (a) and (b), where $\left\{\phi_{i}\right\}$ is a Chebyshev set, the following results.

(i) The sequence $\left\{p_{k}\right\}$ converges to $p^{*}$ which is a best Chebyshev approximation to $f$ on $Z_{1} \subset Z$, where

$$
Z_{1}=\bigcap_{k=1}^{\infty} Y_{k} \text { and } \quad Y_{k}:=\left\{z: w^{k}(z)>0, z \in Z\right\}
$$

$Z_{1}$ contains at least $n+1$ points.

(ii) The sequence $\theta\left(w^{k}\right)=\theta^{k}=\left(\Sigma_{Z} w^{k}(z)\left|f(z)-p^{k}(z)\right|^{2}\right)^{1 / 2}$ is strictly monotonically increasing (unless convergence takes place in a finite number of iterations) and 


$$
\theta^{*}=\lim _{k \rightarrow \infty} \theta^{k}=\max _{z \in Z_{1}}\left|f(z)-p^{*}(z)\right|
$$

(iii) If during the course of the iteration, $w^{k}(z)$ is set to zero for some $z$ (this could happen "accidentally") then, from (i), $Z_{1} \neq Z$ and so we may have $\theta^{*}<$ $\max _{z \in Z}\left|f(z)-p^{*}(z)\right|$, that is, $p^{*}$ is not the best approximation on $Z$. In this case the algorithm can be restarted with the weight function,

$$
\hat{w}(z)=(1-\lambda) \lim _{k \rightarrow \infty} w^{k}(z)+\lambda u(z), \quad 0<\lambda<1,
$$

where $u(z)=1$ for all $z \in Z-Z_{1}$ for which $\left|f(z)-p^{*}(z)\right|>\theta^{*} ; u(z)=0$ otherwise. For $\lambda$ sufficiently small $\hat{\theta}>\theta^{*}$ and after a finite number of restarts, the best approximation to $f$ on $Z$ is obtained.

4. Rate of Convergence. In the real case Lawson [4] and Rice-Usow [7] have observed that $e^{k}$ and $\theta^{k}$ converge linearly to $e^{*}$ and $\left\|e^{*}\right\|$, respectively, with asymptotic convergence factor $\rho^{*}$, where

$$
\rho^{*}=\max \left(\rho=\left|e^{*}(z)\right| /\left\|e^{*}\right\|<1, z \in Z\right) .
$$

Cline [2] has proved that for every $\lambda>\rho^{*}$ there is an $M>0$ such that, for all $k$,

$$
\left\|p^{*}-p^{k}\right\| \leqslant M \lambda^{k} \text { and }\left\|e^{k}\right\|-\left\|e^{*}\right\| \leqslant M \lambda^{k} .
$$

In the complex case some numerical experiments have indicated that the rate of convergence can be slower than linear. For example, we considered the case $f(z)=$ $(\sin z / z)^{1 / 2}$ with $Z$ consisting of 100 points evenly distributed around the boundary of the semidisc $\{z:|z| \leqslant 1, \operatorname{Re}(z) \geqslant 0\}$. Then approximation by a cubic polynomial $\left(\phi_{i}=z^{i-1}\right)$ required $3,6,42$ and $>100$ iterations to obtain, respectively, 1, 2, 3 and 4 correct significant figures in the norm of the error function.

It is possible to extend some of Cline's preliminary results to the complex case (subject to the basic assumption that $\left\{\phi_{i}\right\}$ is a Chebyshev set). For example, his Lemmas 3 and 5 show that at points $z$ not in the extremal set $Z_{0}$, the weights tend to zero as rapidly as a geometric progression with ratio related to $\left|e^{*}(z)\right| /\left\|e^{*}\right\|$. The results of the form (4.1) fail, however, because, with one exception, the algorithm does not converge in one step on pure extremal sets. The exception is the case of extremal sets consisting of only $n+1$ points and is of little value in practice. In fact, as the following example shows, sublinear convergence is possible on pure extremal sets consisting of at least $n+2$ points.

Example. $A:=\{1,-1, i\}, f=z, \phi_{1} \equiv 1$. Here $p^{*}=0$ and $Z_{0}=Z$. If the initial weights at 1 and -1 are equal and the weight at $i$ is nonzero, then

$$
w_{3}^{k+1}=w_{3}^{k} /\left(w_{3}^{k}+\sqrt{\left.1+\left(w_{3}^{k}\right)^{2}\right)}, \quad\left\|e^{k}\right\|=\sqrt{1+\left(w_{3}^{k}\right)^{2}}, \quad\left\|p^{*}-p^{k}\right\|=w_{3}^{k} .\right.
$$

It is clear that there does not exist a constant $\rho<1$ such that

$$
\left\|p^{*}-p^{k+1}\right\| \leqslant \rho\left\|p^{*}-p^{k}\right\| \text { for all } k
$$

Unlike the real case, it is quite possible in practice for $Z_{0}=Z$ (see [5, Chapter 2] 
for examples on the unit disc). In contrast to the above example, our numerical experience with such problems is that the convergence is not generally "very slow". Some examples of this type are presented in Table 1.

5. Acceleration of Convergence. Essentially, we wish to make $w^{k}(z)$ tend to zero as rapidly as possible except at points in some determining set $\widetilde{Z}_{0} \subseteq Z_{0}$ on which $p^{*}$ is also the best Chebyshev approximation to $f$. In the real case various schemes for accelerating this convergence have been proposed ([7], [6]). When $\left\{\phi_{i}\right\}$ is a Chebyshev set, one such scheme recommended in [7] sets values of $w^{k}(z)$ to zero on the basis that $\left|e^{k}(z)\right|$ is "small" in relation to $\left\|e^{k}\right\|$. The success of this scheme depends essentially on the fact that the extremal set (usually $n+1$ points) can be fairly well "regognized" during the course of the iteration. Unfortunately, on the basis of the problems we have treated, this device cannot be recommended in the complex case. Here $\left|e^{k}(z)\right|$ can be close to $\left\|e^{k}\right\|$ for many $z \in Z$; this feature is closely related to the fact that $Z_{0}$ can be "large" in relation to $Z$.

Another modification [7] is to redefine the L1 algorithm by replacing stage (b) with:

$$
\text { Set } w^{k+1}(z)=\frac{\left.w^{k}(z) e^{k}(z)\right|^{2}}{\sum_{Z} w^{k}(y)\left|e^{k}(y)\right|^{2^{2}}}, \quad k=1,2, \ldots, z \in Z .
$$

This scheme, the L2 algorithm, if it converges, makes $w^{k}(z)$ tend to zero like $\left(\rho^{*}\right)^{2 k}$ and so is asymptotically equivalent to two steps of the $\mathrm{Ll}$ algorithm. From (c),

$$
\left(\theta^{k+1}\right)^{2}=\sum_{Z} w^{k+1}(y)\left|e^{k+1}\right|^{2}=\left(\sum_{Z} w^{k}(y)\left|e^{k}\right|^{2}\left|e^{k+1}\right|^{2}\right) /\left(\theta^{k}\right)^{2}
$$

and it is now easy to show (using the Cauchy-Schwartz inequality) that $\theta^{k+1} \geqslant \theta^{k}$, $k=1,2, \ldots$. This is an important part of the convergence proof for the L1 algorithm. Unfortunately, we have observed examples which do not converge (similarly for the real case [4], [7]). When it does converge, however, the acceleration can be quite striking, particularly for examples in which $Z_{0}=Z$. Examples are given in Tables 1 and 2 .

As an effective acceleration scheme for the complex case, we recommend the procedure of applying alternate steps of L1 (stage (b)) and L2 (stage (c)). For this scheme the $\mathrm{L} 3$ algorithm, we have also that $\theta^{k+1} \geqslant \theta^{k}, k=1,2, \ldots$.

Theorem 5.1. Let $\left\{\phi_{i}\right\}$ be a Chebyshev set, and let $f \in C[Z]-P$. Suppose that the positive integers are divided into two sets $I_{1}$ and $I_{2}$ so that if $k \in I_{1}$ step (b) is performed, otherwise step (c) is performed. Given $w^{1} \in W, w^{1}(z)>0$ for all $z \in Z$, then a sufficient condition for the sequence $\left\{p^{k}\right\}$ to converge to $p^{*}$, the best Chebyshev approximation to $f$ on a set $Z_{1} \subset Z$, is that $I_{1}$ be infinite. If $Z_{1} \neq Z$, then the algorithm may be restarted with the restart procedure ((iii), Section 3$)$. The best Chebyshev approximation to $f$ on $Z$ is then obtained after a finite number of restarts.

Proof. The proof that the restart procedure is effective is the same as in the L1 case (a continuity argument plus the fact that $Z$ is finite). Therefore, in order to simplify the proof of the theorem we shall suppose that throughout the iteration no value of $w^{k}(z)$ is accidentally set to zero. We can then deal with best Chebyshev approxi- 
mation to $f$ on $Z$ (as opposed to some subset $Z_{1}$ ).

Using the same argument as in the convergence proof of the L1 algorithm, we can show that $\left\{p^{k}\right\}$ is uniformly bounded. Now consider any convergent subsequence $\left\{p^{k_{i}}\right\}$. Since the $w^{k}$ are also restricted to a compact set, we may assume that $\left\{w^{k_{i}}\right\}$ is also convergent and let $\hat{w}=\lim _{i \rightarrow \infty} w^{k_{i}}$. Using the fact that $\left\{\phi_{i}\right\}$ is a Chebyshev set, we can easily show (as in the L1 convergence proof) that $\hat{w}$ is nonzero at (at least) $n+1$ points. Therefore, in a neighborhood of $\hat{w}$, the best least squares approximation $p$ is a continuous function of the weight $w$; thus $\hat{w}$ corresponds to $\hat{p}$ where $\hat{p}=$ $\lim _{i \rightarrow \infty} p^{k_{i}}$.

Suppose now that $\hat{p} \neq p^{*}$, the best Chebyshev approximation to $f$ on $Z$. By the uniqueness of $\hat{p}, \theta(\hat{w})<\left\|f-p^{*}\right\|$. We shall now examine in $W$ a certain neighborhood of $\hat{w}$ and, by using the fact that $I_{1}$ is infinite, establish a contradiction.

The sequence $\left\{\theta\left(w^{k}\right)\right\}$ is bounded and monotonically increasing, so $\lim _{k \rightarrow \infty} \theta\left(w^{k}\right)$ $=\theta(\hat{w})$. Consider now $T \subset W$, where

$$
T:=\{w:|\theta(w)-\theta(\hat{w})| \leqslant \Sigma, w \in W\}
$$

where $\Sigma=1 / 2 \min \left\{\theta(\hat{w}),\left\|f-p^{*}\right\|-\theta(\hat{w})\right\}$. Then $\theta(w)>0$ for all $w \in T$ and by continuity $T$ is compact. Given $w \in T$ with corresponding best least squares approximation $p$, define the weight function $F(w) \in W$ obtained by one step of the L1 algorithm

$$
F(w)(z)=\frac{w(z)|f(z)-p(z)|}{\sum_{y \in Z} w(y)|f(y)-p(y)|}, \quad z \in Z
$$

and let $\delta(w)=\theta(F(w))-\theta(w)$. Then, $\delta(w) \geqslant 0$ for all $w \in T$; also, since $p$ is continuous on $T, F$ is continuous on $T$ so there exists $w_{0} \in T$ such that $\delta=\delta\left(w_{0}\right)=$ $\inf _{w \in T} \delta(w)$. Since the L1 algorithm is convergent ((i), (ii), Section 3), a weight function $w^{*}$ corresponding to $p^{*}$ satisfies $\theta\left(w^{*}\right)=\left\|f-p^{*}\right\|$ and $F\left(w^{*}\right)=w^{*}$. Consequently, $w_{0}$ cannot be such a $w^{*}$; and hence, $\delta>0$. We have thus shown that for any $w \in T$ one step of the L1 algorithm must increase the least squares error by at least $\delta$.

Now consider the original sequence $\left\{\theta\left(w^{k}\right)\right\}$ (generated by the algorithm) along with the subsequence $\left\{\theta\left(w^{k_{i}}\right)\right\}$. Choose $K$ such that, for $k \geqslant K,\left|\theta\left(w^{k}\right)-\theta(\hat{w})\right|<$ $\min (\delta / 2, \Sigma)$. Since $I_{1}$ is infinite, we may also choose $k_{0} \in I_{1}$ with $k_{0}>K$, so that for $i$ sufficiently large

$$
\theta\left(w^{k}\right) \geqslant \theta\left(w^{k} 0^{+1}\right)=\theta\left(F\left(w^{k} 0\right)\right)
$$

Since $w^{k} 0 \in T$

$$
\theta\left(w^{k} i\right) \geqslant \theta\left(w^{k} 0\right)+\delta>(\theta(\hat{w})-\delta / 2)+\delta>\theta(\hat{w})
$$

but this contradicts the fact that $\theta\left(w^{k}\right)$ tends to $\theta(\hat{w})$ from below.

We have thus shown that every convergent subsequence of $\left\{p^{k}\right\}$ tends to the best approximation $p^{*}$. Since $\left\{p^{k}\right\}$ is uniformly bounded this is sufficient to guarantee that $p^{k} \rightarrow p^{*}$ as $k \rightarrow \infty$, which completes the proof.

6. Computational Details. Numerical Examples. For the three algorithms L1, L2 and L3 we have not observed any examples in which values of $w^{k}(z)$ are accidentially set to zero. (Rice and Usow [7] report for the L1 algorithm, in the real case, that only very rarely is it necessary to use the restart procedure.) 
TABLE 1

Approximation on the unit disc (100 points on the boundary). All the points are extremal points, $Z_{0}=Z ; \phi_{i}=z^{i-1}, i=1,2, \ldots, n$.

\begin{tabular}{cccccc}
\hline$f(z)$ & $n$ & $\begin{array}{c}\text { Correct } \\
\text { significant } \\
\text { figures }\end{array}$ & $\begin{array}{c}\text { L1 } \\
\text { iterations }\end{array}$ & $\begin{array}{c}\text { L2 } \\
\text { iterations }\end{array}$ & $\begin{array}{c}\text { L3 } \\
\text { iterations }\end{array}$ \\
\hline \multirow{2}{*}{$e^{z}$} & 5 & 3 & 9 & 2 & 3 \\
& 7 & 6 & 17 & 3 & 5 \\
& 9 & 6 & 18 & 3 & 5 \\
& 11 & 5 & 14 & 3 & 4 \\
\hline & 3 & 5 & 19 & 2 & 5 \\
& 4 & 6 & 19 & 2 & 5 \\
$z-2$ & 7 & 5 & 18 & 2 & 5 \\
& 9 & 6 & 20 & 2 & 5 \\
& 11 & 4 & 14 & 2 & 3 \\
\hline
\end{tabular}

TABLE 2

Approximation on $\{z:|z| \leqslant 1, \operatorname{Re}(z) \geqslant 0\}$ (100 points on the boundary), $Z_{0} \neq Z$, $\phi_{i}=z^{i-1}, i=1,2, \ldots, n$.

\begin{tabular}{lccccc}
\hline$f(z)$ & $n$ & $\begin{array}{c}\text { Correct } \\
\text { significant } \\
\text { figures }\end{array}$ & $\begin{array}{c}\text { L1 } \\
\text { iterations }\end{array}$ & $\begin{array}{c}\text { L2 } \\
\text { iterations }\end{array}$ & $\begin{array}{c}\text { L3 } \\
\text { iterations }\end{array}$ \\
\hline$e^{z}$ & 7 & 1 & 5 & 2 & 3 \\
& 11 & 1 & 3 & 2 & 3 \\
\hline$\frac{1}{\sqrt{(2 z+1)}}$ & 5 & 2 & 59 & 34 & 39 \\
\hline $\sin \sqrt{z}$ & 9 & 2 & 12 & 7 & 8 \\
$\sqrt{z}$ & 4 & 1 & 4 & 3 & 5 \\
\hline
\end{tabular}


TABLE 3

Rational approximation with preassigned poles. Approximation of $f(z)=$ $\left[1+(z+1)^{2}\right]^{-1 / 2}$ using 25 points on $\{z: z=i y,-20 \leqslant y \leqslant 20\}$. The letter $G$ indicates that more than 30 iterations we required.

\begin{tabular}{|c|c|c|c|}
\hline$j=1,2, \ldots, n$ & $n$ & $\begin{array}{l}\text { Correct } \\
\text { significant } \\
\text { figures }\end{array}$ & $\begin{array}{c}\text { L3 } \\
\text { iterations }\end{array}$ \\
\hline \multirow{12}{*}{$\frac{1}{(1+z)^{j-1}}$} & \multirow{3}{*}{4} & 1 & 2 \\
\hline & & 2 & 5 \\
\hline & & 3 & 26 \\
\hline & \multirow{3}{*}{5} & 1 & 3 \\
\hline & & 2 & $G$ \\
\hline & & 3 & $G$ \\
\hline & \multirow{3}{*}{6} & 1 & 3 \\
\hline & & 2 & 5 \\
\hline & & 3 & $G$ \\
\hline & \multirow{3}{*}{7} & 1 & 5 \\
\hline & & 2 & $G$ \\
\hline & & 3 & $G$ \\
\hline \multirow{12}{*}{$\frac{1}{(z+j)}$} & \multirow{3}{*}{4} & 1 & 2 \\
\hline & & 2 & 27 \\
\hline & & 3 & $G$ \\
\hline & \multirow{3}{*}{5} & 1 & 1 \\
\hline & & 2 & 7 \\
\hline & & 3 & 12 \\
\hline & \multirow{3}{*}{6} & 1 & 2 \\
\hline & & 2 & 15 \\
\hline & & 3 & $G$ \\
\hline & \multirow{3}{*}{7} & 1 & 5 \\
\hline & & 2 & 17 \\
\hline & & 3 & $G$ \\
\hline
\end{tabular}


The recommended L3 algorithm consisting of alternate steps of L1 and L2 has been applied successfully to many problems. Some results are presented in the tables and comparisons are made in Tables 1 and 2. For each case, $w^{1}(z)=1 / N$ for all $z \in$ $Z$. The weighted least squares problems were solved by using the complex analogue of the Golub algorithm [3]. In the tables we have indicated the number of iterations required for a certain number of correct significant figures in the error norm; this was made possible by using the inclusion interval

$$
\theta^{k} \leqslant\left\|f-p^{*}\right\| \leqslant\left\|f-p^{k}\right\|
$$

for the final computed iteration.

It is also interesting to compare the behavior of the L1 and L3 algorithms when applied to the example of Section 4, for which some computed results are as follows.

$$
\begin{aligned}
& \left\|p^{*}-p^{5}\right\|=0.1342 \text { (L1), } 0.0956 \text { (L3); } \quad\left\|e^{5}\right\|=1.0090 \text { (L1), } 1.0046 \text { (L3), } \\
& \left\|p^{*}-p^{50}\right\|=0.0187 \text { (L1), } 0.0125 \text { (L3); } \quad\left\|e^{50}\right\|=1.000175 \text { (L1), } 1.000078 \text { (L3). }
\end{aligned}
$$

Given that a complex least squares routine is available, the L3 algorithm is easily programmed. It must be accepted, however, that the ultimate convergence of the algorithm is in general very slow; but often good approximations are obtained in a small number of iterations. We are not aware of an algorithm which is faster.

7. Acknowledgment. S. Ellacott wishes to thank the Science Research Council for their support in providing a Research Studentship. Also the authors wish to thank the referee for his valuable suggestions and in particular for providing the example in Section 4.

\footnotetext{
Department of Mathematics

Brighton Polytechnic

Brighton BN2 4GJ, England

Department of Mathematics

University of Manchester

Manchester M13 9PL, England
}

1. E. W. CHENEY, Introduction to Approximation Theory, McGraw-Hill, New York, 1966. MR 36 \#5568.

2. A. K. CLINE, "Rate of convergence of Lawson's algorithm," Math. Comp., v. 26, 1972, pp. 167-176. MR $45 \# 7921$.

3. G. H. GOLUB, "Numerical methods for solving linear least squares problems," Numer. Math., v. 7, 1965, pp. 206-216. MR 31 \#5323.

4. C. L. LAWSON, Contributions to the Theory of Linear Least Maximum Approximations, Thesis, UCLA, 1961.

5. G. G. LORENTZ, Approximation of Functions, Holt, Rinehart and Winston, New York, 1966. MR $35 \# 4642$; erratum, 36, p. 1567.

6. J. R. RICE, The Approximation of Functions. Vol. 2: Nonlinear and Multivariate Theory, Addison-Wesley, Reading, Mass., 1969. MR 39 \#5989.

7. J. R. RICE \& K. H. USOW, "The Lawson algorithm and extensions," Math. Comp., v. 22, 1968, pp. 118-127. MR $38 \# 463$.

8. T. J. RIVLIN \& H. S. SHAPIRO, "A unified approach to certain problems of approximation and minimization," J. Soc. Indust. Appl. Math., v. 9, 1961, pp. 670-699. MR 24 \#A3462.

9. J. WILliAMS, "Numerical Chebyshev approximations in the complex plane," SIAM J. Numer. Anal., v. 9, 1972, pp. 638-649. MR 47 \#2784. 